\title{
Construyendo ciudadanía en la universidad: Una propuesta para la convivencia y resolución de conflictos
}

\section{Building citizenship in the university: A proposal for coexistence and conflict resolution skills}

\author{
Fecha de recepción: \\ 30 Agosto del 2021 \\ Chávez Ponce Daniel Fernando ${ }^{1}$ \\ y Norzagaray Benítez Claudia Cecilia ${ }^{2}$ \\ Fecha de aprobación: \\ o3 Noviembre del 2021

\begin{abstract}
Autor de correspondencia. Licenciado en Psicología, especialidad área educativa y Maestría en Innovación Educativa Américas.

Correo: ps.edu.dfcp@gmail.com. ORCID: https://orcid.org/oooo-ooo2-7261-6897 el Centro de Investigación en Educación Virtual y Doctorado en Educación en la Universidad Nacional de Educación a Distancia. Actualmente profesora de tiempo completo del departamento de Psicología y Ciencias de la Comunicación de la Universidad de Sonora.

Correo: cecilia.norzagaray@unison.mx. ORCID: https://orcid.org/oooo-ooo3-4695-112X
\end{abstract} \\ ambas en la Universidad de Sonora. Actualmente profesor de asignatura en Universidad Kino y Universidad Vizcaya de las \\ ${ }^{2}$ Licenciada en Psicología, especialidad área clínica en la Universidad de Sonora; Maestría en Desarrollo Educativo en
}

\section{Resumen}

Las Instituciones de Educación Superior tienen el compromiso de formar integralmente al estudiantado, por lo que no es posible aludir a una educación de calidad si no se contempla la formación de competencias ciudadanas. Estas se agrupan en tres ámbitos: convivencia y paz, participación y responsabilidad democrática y pluralidad, identidad y valoración de diferencias. Dado que el conflicto es inevitable que se presente en la interacción de los individuos, es relevante desarrollar habilidades que permitan resolverlos de manera pacífica. Por ello, el presente trabajo presenta una propuesta para promover competencias ciudadanas a través de la convivencia y la paz, enseñando la resolución de conflictos a partir de la promoción de habilidades para la colaboración. La propuesta se estructuró a partir de dos aspectos: un diagnóstico de necesidades en una muestra de estudiantes universitarios, y teóricamente se retoma el enfoque constructivista, teniendo como método el aprendizaje colaborativo de las que se derivan estrategias didácticas creativas, significativas e integradoras. La propuesta tiene por objetivo el desarrollo de habilidades para resolver conflictos de manera colaborativa, el manejo de las emociones, la mejora de las relaciones interpersonales y asumir el rol de liderazgo sin autoritarismo, aspecto fundamental para el desempeño del estudiante y su futuro rol en la sociedad. Se concluye que es necesario que las universidades ofrezcan a los estudiantes instancias y escenarios para promover la participación y la interacción con los diferentes actores educativos, con el fin principal de construir ciudadanía.

Palabras clave: formación ciudadana, convivencia, resolución de conflictos, universitarios.

\section{Abstract}

Higher Education Institutes are committed to offer a well-rounded education for the student body, so it's not possible to refer to a quality education if the citizenship skills are not contemplated. These are grouped into three areas: coexistence and peace, participation and democratic responsibility and plurality, identity, and valuation of differences. Since conflict is unavoidable in the interaction of individuals, it's relevant to develop emotional skills that allow them to be resolved peacefully. For this reason, this paper presents a proposal to promote civic competences through coexistence and peace, teaching conflict resolution skills by collaboration. 
The proposal was structured in a constructivist approach, having as a method collaborative learning, from which derives creative, meaningful, and integrative didactic strategies. The proposal has for objective the development of skills to solve conflicts collaboratively, managing emotions, improving interpersonal relationships and assuming the leadership role without authoritarianism, a fundamental aspect for student performance and their future role in society. It's concluded that is necessary for universities to offer the student body instances and scenarios to promote participation and the interaction between the school community, so citizenship can be build.

Keywords: Education Citizenship, coexistence, resolution skills, university students

\section{Introducción}

Las Instituciones de Educación Superior (IES), tienen un gran compromiso de formar de manera integral a los jóvenes profesionistas, esto quiere decir, habilitarlos para que sean capaces de resolver problemas, pero que además desarrollen competencias genéricas que le permitan desenvolverse no solo en el plano profesional, sino también en el personal y social.

La Formación Ciudadana (FC) es una parte integral de este proceso formativo ya que además de considerarse una habilidad para la vida, a través de ella se fortalecen las actitudes, valores y habilidades para la relación del individuo con su entorno, delegándole un rol activo, participativo y dinámico en la universidad y la sociedad. No es suficiente contar con un buen desempeño académico para considerarse un buen ciudadano, sino que deben realizarse desempeños activos, transformadores y críticos (Venet, 2019).

Gran parte de los problemas de hoy se centran en la falta de respeto a la diversidad, a la exclusión, la intolerancia y la violencia. Por tanto, es necesario asumir una postura opuesta a estas actitudes que van en contrasentido de las políticas públicas expresadas por diversos organismos como UNESCO (2015) e incluso en los planes nacionales de los países como México 2019-2024 (PND, 2019). Como expresa Martínez (2006) no podemos aludir a una formación universitaria de calidad, si no se consideran aprendizajes sobre y para la ciudadanía.
La FC a nivel universitario tiene presencia predominanteen losdocumentosinstitucionales, sin embargo, existen carencias de acciones formativas reales. Esto es porque, como argumenta León (2020), a la universidad se le ha otorgado la función de desarrollar conocimiento a nivel disciplinar y práctica para la solución de problemas, más que en los componentes actitudinales. La construcción de la ciudadanía no sucede de manera natural y las IES deben generar espacios de interactividad para formar a los estudiantes para convivir con otros, respetando las diferencias individuales, los derechos humanos y ejercer la ciudadanía. Por tanto, se entiende por formación ciudadana "el saber técnico disciplinar, al servicio del desarrollo de la comunidad" (p. 377), es decir, todo proceso que enseñe al individuo a vivir en comunidad, con la finalidad de que se integre y participe considerando elementos como el compromiso, la comunicación, conocimiento de obligaciones y derechos, así como el ejercicio de estos.

En el nivel universitario, implica difundir, defender y ejercer los derechos humanos como base de una convivencia pacífica, para participar en la vida política y comprender de manera crítica a la sociedad, sus instituciones y normas. Es relevante que los estudiantes universitarios asuman valores que permitan el ejercicio de prácticas y la consecución de normas que coadyuven a la mejora de sus interacciones cotidianas, que se propician en las instituciones educativas, procurando que estos se involucren en los procesos de toma de decisiones, elaboración de normas, conocimiento de los principales problemas de la comunidad educativa, para que de esta manera puedan participar de manera pacífica para mejorar la convivencia (Ortiz y Lemus, 2020).

El ejercicio de la ciudadanía en y desde la escuela implica diversos retos. Para ello, el Ministerio de Educación Nacional (MEN, 2011) de Colombia ha propuesto competencias ciudadanas, a las cuales las concibe como la integración de actitudes, conocimientos, emociones, cogniciones para ser sujetos participativos. El estudio de Mescua, Ramos, Ramírez, Cruz y Caycho (2020) en estudiantes universitarios muestra que obtienen valores promedio correspondientes a un nivel regular, expresando que requieren enfatizar las estrategias de aprendizaje y el desarrollo de habilidades emocionales. Consideran que, en la actualidad, la docencia no solo media conocimientos sino elementos emocionales. 
$\mathrm{Al}$ indagar sobre las competencias deseables en los futuros egresados por la universidad, se descubrió un consenso en relación con los conocimientos para el ejercicio de la ciudadanía como la conciencia intercultural, las posibles consecuencias en la toma de decisiones, el desarrollo de la empatía, la reflexión y el pensamiento crítico, así como el desarrollo de habilidades emocionales como la asertividad, el diálogo constructivo, la resolución de conflictos, el manejo de las emociones, etc. (Hamra, 2020), elementos fundamentales para los cimientos de las competencias participativas y democráticas.

Las competencias ciudadanas se ubican en tres ámbitos: 1) el convivir de manera pacífica y constructivamente con otros que pueden tener intereses, fines $\mathrm{o}$ metas diferentes $\mathrm{u}$ opuestas, 2) la elaboración de acuerdos y consensos sobre normas y decisiones que regulan a las personas y que deben favorecer el bien común, 3) el ejercicio de la ciudadanía en sí mismo, lo que conlleva a construir una sociedad a partir del reconocimiento basado en el respeto mutuo. En este sentido, las competencias ciudadanas son las capacidades que ayudan a enfrentar constructivamente cada uno de estos retos (Murillo y Castañeda, 2007; Ruiz y Chaux, 2005; MEN, 2014.).

Para los objetivos de este trabajo nos centraremos en el ámbito de formación desde la convivencia y la paz, la cual se entiende cómo la capacidad de trabajar con otros, de resolver las diferencias, así como los conflictos que se presentan en la interacción escolar de las instituciones educativas (Fierro, 2011, en Verdeja, 2013). Estos elementos van de la mano pues la convivencia proporciona el componente práctico y la armonía social, a la democracia en el ambiente escolar, ya que favorece el trabajo colaborativo por medio del respeto y sin discriminación, la ayuda mutua, la promoción de los derechos humanos, el diálogo, la valoración y aceptación del otro, inclusive la conciencia ecológica en la interacción con el entorno (Montoya, 2008).

Para Chaux (2012) son ocho competencias para una convivencia pacífica, las cuales son el manejo de ira, empatía, toma de perspectiva, generación de opciones, consideración de consecuencias, pensamiento crítico, escucha activa y asertividad. Tres de ellas se ligan a las emociones (manejo de ira, empatía y asertividad) el resto son competencias integradoras que coadyuvan a la dirección de las motivaciones emocionales siendo un contrapeso cognitivo de las emociones. Con ello, nos muestra que al formar ciudadanía se construye convivencia y seguridad sostenible a través de la reducción de agresiones, resolución de conflictos escolares y la reducción de la intimidación escolar.

Al respecto, Chaux (2012, citado en Zuta, Velasco y Rodríguez, 2014) plantea cinco principios pedagógicos para el logro de aprendizajes ciudadanos:

1. Aprender haciendo:Aludeaquela resolución de conflictos se aprende resolviendo las diferencias e intentarlo cuando esto sea necesario y no porque nos platiquen de ello.

2. Aprendizaje significativo: Se refiere a diseñar situaciones en las que los estudiantes lo relacionen con la vida real y puedan encontrar un significado y utilidad en su entorno, esto puede ser a través de situaciones simuladas o reales.

3. Incrementar la complejidad de los aprendizajes de manera progresiva: durante la enseñanza de las competencias ciudadanas deben presentarse cada vez con mayor complejidad y retos, pero que sean solubles y alcanzables.

4. Autoeficacia: Proporcionar a los estudiantes la seguridad de que son capaces de resolver problemas que se les presentan de manera cotidiana, por lo que se vincula de manera estrecha en el punto anterior.

5. Motivación intrínseca: es relevante que los estudiantes valoren el uso de las habilidades en su vida cotidiana y no sean obligados a hacerlo; deben percibir los beneficios para su vida, relacionarse con los otros y con compromiso de una sociedad más justa.

Uno de los objetivos de la FC es que los individuos convivan de manera pacífica y constructiva, lo cual no implica que siempre prevalezca la armonía o la ausencia de conflictos, pues en un grupo social en que el que existen intereses, metas e ideales diversos es complejo que no existan desacuerdos con otros. Más bien, lo que se solicita es que los conflictos sean resueltos sin agresión y en beneficio de las partes involucradas y que las personas hagan valer sus derechos y los de otros y que acudan a instancias reguladoras cuando así se requiera (Mejía y Perafán, 2006). 
Unconflictosepresentacuandohayincompatibilidad entre las personas, quienes dadas sus diferencias no logran alcanzar acuerdos, lo cual lo hace propio de las personas que interactúan con otros (Pinilla y Mendieta, 2013). Los conflictos no deben valorarse como algo negativo sino como situaciones potenciales de aprendizaje y del desarrollo de los individuos en la sociedad, recuperando la posibilidad de emplearlo para promover diversos aprendizajes que coadyuven a la formación del estudiantado, generando espacios para la reflexión (Baldovino y Reyes, 2017).

Ante esto, es de suma importancia reconocer las habilidades que tienen los estudiantes para resolver los conflictos y que se desarrollan cotidianamente en la convivencia escolar, conocidos como Estilos de Resolución de Conflictos, los cuales se definen como la tendencia del individuo a comportarse ante la presencia de una incompatibilidad en relación con el pensamiento, comportamiento, creencia o actitud de otra persona, para encontrar soluciones que satisfagan a las partes involucradas (Holst, Galicia, Gómez y Degante, 2017).

Thomasy Kilmann(2014) realizanunacategorización de estos estilos, a través de la intersección de dos variables: 1. La asertividad, entendida como la habilidad con la que la persona busca solucionar los conflictos y, 2. La colaboración, que hace referencia a la intención de resolver conflictos con el apoyo de otros individuos. A partir del nivel en el que se presenta en los individuos, se establecen cinco variantes:

1. Competidor: Corresponde a un nivel alto de asertividad, pero un nivel bajo de colaboración, pues la persona actúa para satisfacer su propio interés, a expensas de la otra persona involucrada.

2. Colaborador: Corresponde a un equilibrio entre la asertividad y la colaboración. Podría considerarse como el estilo esperado en todos los individuos, pues se basa en el trabajo en equipo para resolver, en conjunto, el conflicto que se presenta en las partes involucradas, explorando el desacuerdo y buscando soluciones creativas (Luna, 2018).

3. Comprometido: Corresponde a un nivel intermedio entre la asertividad y la colaboración, pues se centra en buscar una solución oportuna y mutuamente aceptable, estableciendo un compromiso entre las dos partes.
4. Evasivo: Existencia nula de asertividad y colaboración, ya que el individuo evade la situación del conflicto, no habla de ello, aplaza su posible solución, o simplemente se aparta.

5. Complaciente: Corresponde a un nivel bajo de asertividad y un nivel alto de colaboración. El individuo complace los deseos e intereses de la otra parte involucrada, obedeciendo órdenes, además de ser generoso o altruista.

Carreño y Rozo (2020) realizan un análisis sistemático para identificar las estrategias que se han desarrollado para la favorecer el desarrollo de la convivencia y la paz desde la educación, organizándose en cuatro: 1) prevención, las cuales reúnen acciones para manejar emociones, fortalecer valores y prácticas como el diálogo, la tolerancia y el respecto, 2) de participación, incluyen proyectos para sensibilizar a los estudiantes frente a su responsabilidad democrática, para ello, organizan proyectos pedagógicas como asambleas, 3) de intervención, en las cuales se toman acciones para mitigar los actos de violencia, y 4) el uso de tecnologías, las cuales son transversales potenciando la creación de espacios tecnológicos y espacios virtuales en relación a la paz en sus instituciones.

En consideración de los planteamientos acerca de la resolución de conflictos como un conjunto de habilidades para mejorar la convivencia escolar y la paz que se presenta en las instituciones educativas y como base para la participación de los estudiantes en su entorno educativo, se presenta el diseño de un programa para la promoción de resolución de conflictos a través del diálogo, la colaboración, la empatía, el liderazgo, así como la disposición afectiva de los individuos; la propuesta se basa en los principios pedagógicos del constructivismo y del aprendizaje colaborativo.

\section{Fundamentación de la propuesta}

Para la formulación de la propuesta se consideraron dos aspectos: 1. Una evaluación de los estilos de resolución de conflictos, así como, la descripción de la percepción de la convivencia escolar de la IES y 2. Una vez identificados los aspectos a mejorar, se realizó la fundamentación teórica, con el fin de comprender la mejor manera de enseñar estas habilidades a los estudiantes universitarios. A continuación, se presentan ambas. 


\section{Evaluación de la convivencia escolar y la resolución de conflictos}

Se realizó un diagnóstico de necesidades a partir de un método de investigación mixto, tomando en cuenta datos cuantitativos que permitan generalizar a otras poblaciones, además de profundizar en dichos datos a partir de la descripción de las percepciones de los estudiantes con respecto a la convivencia y la resolución de conflictos, aumentando la comprensión del objeto de estudio (Cresswell, 2009).

Desde el enfoque cuantitativo se implementó, a través de un formulario en la plataforma "Google Forms", el instrumento "Modos de Manejo de Conflictos" de Thomas y Kilmann (2014), con lo cuál se busco determinar el estilo de resolución de conflictos que predominaba. El análisis de los datos se realizó a través del programa Statiscal Package for Social Sciences (SPSS), ubicando los puntajes brutos derivados de las respuestas de los estudiantes para estudiar los porcentajes de cada uno. Se encontró que el estilo que predomina es el comprometido, así como la evasión de la situación conflictiva; por su parte, la colaboración queda ubicada en rango medio bajo, indicando que los estudiantes no suelen trabajar en equipo para resolver la situación que aqueja en la convivencia escolar.

Desde el enfoque cualitativo se llevó a cabo un grupo focal para describir la percepción de convivencia escolar y la solución de conflictos en la Institución de Educación Superior, desde las experiencias del estudiantado. El grupo se realizó a través de la plataforma "Microsoft Teams" y tuvo una duración de 2 horas. Para el análisis de los datos se empleó el programa Atlas.Ti en su versión 9, y se organizaron dos grandes categorías de análisis: convivencia escolar y resolución de conflictos. Respecto a la primera, los estudiantes perciben una convivencia agradable entre compañeros, y algunos docentes, gracias a los espacios que proporciona la institución para la interacción, sin embargo, consideran necesario la accesibilidad de los protocolos para resolver las situaciones conflictivas, así como de actividades extracurriculares que los ayuden a participar en la mejora de su institución; sobre la segunda, los estudiantes resaltaron la influencia de los estados afectivos (emociones y sentimientos) en la generación de un conflicto, así como la falta de habilidades para el liderazgo al momento de trabajar en equipo, entre otros; Los factores que generan conflictos entre ellos y profesores fueron las diferencias en pensamientos, creencias e ideas sobre ciertos problemas sociales (Chávez, 2021).

Se concluye que los estudiantes necesitan ser participes de actividades extracurriculares que apoyen a la construcción de mejores habilidades que les permitan convivir de una mejor manera con los demás integrantes de las instituciones educativas, así como, proporcionar soluciones efectivas y eficientes a los conflictos que se presenten, tomando en cuenta aspectos afectivos, de liderazgo, relaciones interpersonales, así como los principales elementos que generan conflictos. Aportando a la formación integral del estudiante, así como a las condiciones para la construcción de la ciudadanía en las IES.

\section{Enfoque y método de aprendizaje}

Los fundamentos del modelo constructivista ofrecen un sustento que permiten integrar elementos pertinentes en el proceso de enseñanza-aprendizaje. Su fundamento principal se basa en la importancia de las relaciones sociales que el estudiante establece en las instituciones educativas, las cuales ayudan a fortalecer y perfeccionar las habilidades que ha desarrollado durante su formación académica (Schunk, 2009).

Busca empoderar al estudiante para que este se haga cargo de sus propios procesos de aprendizaje, a partir de la guía de un docente, lo cual apremia el desarrollo del pensamiento crítico, propiciando un proceso de reconstrucción entre lo nuevo que está aprendiendo y lo que ya conoce sobre el tema (Aparicio y Ostos, 2018). Por lo tanto, la función del docente se enfoca en la organización sistemática de cada uno de los contenidos didácticos, promoviendo las condiciones para lograr un desarrollo efectivo de los aprendizajes esperados de los estudiantes.

De acuerdo con Ortiz e Hincapié (2019), la organización del contenido didáctico debe de responder a cuatro aspectos fundamentales: 1) cada tema y actividad deberá de mantener una secuencia lógica; 2) motivará al estudiante a participar; 3) los contenidos estarán enfocados en la habilidad que se pretende enseñar, y, 4) hacer explícitas las características principales que componen el aprendizaje esperado. Estos aspectos ofrecen una visión distinta a lo que podríamos considerar tradicionalmente como el aprendizaje, 
en donde se buscaría establecer una funcionalidad de las habilidades que se desarrollan en las sesiones didácticas con el fin de ofrecer una perspectiva realista de lo que se está aprendiendo, apelando a las situaciones que podría vivir el estudiante.

El modelo constructivista proporciona la suficiente evidencia para facilitar la comprensión de estructurar escenarios donde la socialización sea el foco central de atención, a diferencia de los modelos tradicionales los cuales ya no se ajustan a las condiciones y necesidades actuales de los estudiantes. Es importante destacar que las instituciones educativas estructuran diversos espacios de socialización en donde los estudiantes pueden interactuar entre sí, por lo tanto, estos lugares deben de ser tomados en cuenta para el proceso de enseñanza-aprendizaje, pues el socializar los conocimientos relacionados a la formación profesional, a través del diálogo, permite dar un significado al aprendizaje desarrollado.

En este sentido, integrar el aprendizaje colaborativo como un método de enseñanza alternativo al tradicional, apoyaría a la idea constructivista de ofrecer las condiciones apropiadas para la socialización del conocimiento. Al estructurar una secuencia didáctica, con base a esta idea, se toman en cuenta las siguientes características (Johnson, Johnson y Holubec, 1999, en Iborra e Izquierdo, 2010):

1. La interdependencia positiva. Derivar metas y propósitos en compañía de los estudiantes, que ayude a definir la identidad y reconocimiento del grupo, ofreciendo la guía necesaria a los estudiantes en la división estratégica de los recursos con los que cuentan en el aula, especificando el rol y el trabajo que a cada uno le toca realizar durante su estancia en la institución educativa.

2. Las interacciones cara a cara. Promover la interacción de los estudiantes a través de técnicas grupales, donde colaboren entre sí para alcanzar los objetivos previstos por el docente, propiciando la empatía, solidaridad, respeto y el esfuerzo.

3. Responsabilidad individual. Establecer actividades dónde el estudiante determine sus propios alcances y limitaciones, apoyándolo cuando sea necesario a superar aquellos obstáculos que él considera importante para su aprendizaje, reforzando también aquellos conocimientos que ha desarrollado.

4. Enfatizar el desarrollo de las habilidades sociales. Potenciar comportamientos como la comunicación, la resolución de conflictos, la negociación, el liderazgo, la asertividad, entre otros, promoviendo la expresión de ideas entre los estudiantes.

5. Autorreflexión grupal. Ofrecer un espacio de reflexión, al finalizar la sesión, para que el grupo de estudiantes analicen y comparen los aprendizajes previos a la sesión con los nuevos que han desarrollado, identificando y describiendo las fortalezas y áreas de oportunidad que se habrían de atender.

Bajo esta lógica, las secuencias didácticas estructuradas para el programa educativo deberán de promover la interacción constante entre los estudiantes, para ello, las estrategias de aprendizaje acorde para la promoción de aprendizajes desde esta perspectiva son: los juegos interactivos, pues permite el desarrollo de las habilidades de colaboración en los escenarios de educación, contando con actividades que promuevan la reflexión, la comprensión, y el análisis de los contenidos de la secuencia didáctica (Padilla, 2011).

El juego de rol también propicia la participación del estudiante, a través de la interpretación de situaciones ligadas a la realidad de los individuos, explicitando las características de los sujetos que los participantes interpretarán, brindando la lógica necesaria para representar la habilidad que se busca que se desarrolle (Urbina, Medina y Calle, 2010; Holguín, 2018). Por otro lado, los estudios de caso coadyuvan al desarrollo del pensamiento crítico de los estudiantes, a través del análisis de situaciones reales, lo que permite al estudiante comprender el origen de diversos problemas, las variables involucradas, así como las posibles soluciones que pueden brindar de acuerdo con su conocimiento (Tamayo, 2011).

Fomentar la participación de los estudiantes en debates, foros de discusión, así como la creación de talleres didácticos y grupos de estudio para la promoción de habilidades, ubicados dentro de una problemática común que se enfrentan en su institución educativa, concientiza a los estudiantes 
en la importancia del trabajo en equipo y la colaboración entre pares para alcanzar objetivos en común (Scagnoli, 2006; Betancourt, Guevara y Fuentes, 2011; Andrés y Labrador, 2014). Con base a esta lógica analítica, se elaboró un programa de innovación educativa, que busca formar a los estudiantes universitarios en habilidades para la colaboración, al momento de ofrecer soluciones a los conflictos de la comunidad educativa.

\section{ConVive: Aprende a resolver conflictos colaborando con los demás}

A fin de buscar la promoción de la formación integral de los estudiantes, se diseñó un programa de innovación educativa, para propiciar el desarrollo de habilidades de resolución de conflictos en estudiantes universitarios, cuyo propósito principal es la mejora de la convivencia escolar en las Instituciones de Educación Superior. Para ello, se retoma la propuesta de Torrecillas, Martínez, Olmos y Rodríguez (2016), quienes facilitan la comprensión del orden lógico que se habría que tomar en cuenta para la formación de dichas habilidades:

1. Identificación de conflictos y estilos para resolverlos.

2. Gestión de emociones personales durante el conflicto.

3. Gestión de las relaciones interpersonales dentro de la institución educativa.

4. Potenciación de climas colaborativos en la institución educativa.

La propuesta se divide en 8 sesiones de trabajo (dos sesiones para cada unidad) cuya duración oscila entre una hora y media a dos horas, dónde, a través de juegos de roles, estudios de caso, juegos interactivos, y la participación en foros, los estudiantes desarrollan los aprendizajes esperados que se describen a continuación:

1. Identifica los elementos que constituyen un conflicto en la convivencia escolar universitaria, a través de un juego de rol, en un espacio de reflexión acerca de las situaciones conflictivas que se viven en la convivencia escolar.

2. Describe las características del estilo colaborativo de resolución de conflictos, a través de un estudio de caso, enunciando alternativas de solución para situaciones conflictivas que se presentan en la convivencia escolar.

3. Interpreta las sensaciones que experimenta su cuerpo al enfrentarse a un conflicto, a través de un juego interactivo, mencionando los sentimientos experimentados.

4. Practica las técnicas de regulación y expresión de estados afectivos de Steven Hayes (Di que sí, Juego imposible, Meditación Sencilla y Moverse en cámara lenta), a través de un juego interactivo, para establecer habilidades que permitan el control efectivo de emociones y sentimientos.

5. Analiza los intereses y desinterés de sus compañeros de aula, a través de un estudio de caso, que propicie relaciones interpersonales positivas dentro de la comunidad educativa.

6. Selecciona las características principales que le permiten confiar en sus compañeros de aula, a través de un juego de roles, para distinguir las acciones que promueven una mejor convivencia escolar.

7. Expone las características personales que lo convierten en un líder al momento de colaborar con otros compañeros, a través de un juego interactivo, que establece mejores climas colaborativos entre los estudiantes de la comunidad universitaria.

Cada actividad es monitoreada y evaluada a través de instrumentos cualitativos como lo son: las listas de cotejo, las escalas de estimación y las rúbricas de evaluación, permitiendo conocer el proceso cognitivo que los estudiantes han tenido que transcurrir para la construcción de su aprendizaje.

Para la evaluación del programa se propone la utilización de una batería de instrumentos (ver Tabla 1), la cual permite medir cuantitativamente las habilidades de los estudiantes antes y después de la implementación, obteniendo información relevante con respecto a la funcionalidad del programa para promover y desarrollar alternativas de solución de conflictos en estudiantes universitarios. 
Tabla 1. Batería de instrumentos para la preevaluación y post evaluación de los participantes del programa: "ConVive"

\begin{tabular}{|c|c|c|c|}
\hline $\begin{array}{l}\text { Nombre del } \\
\text { instrumento }\end{array}$ & Autor & Dimensiones & $\begin{array}{l}\text { Cantidad } \\
\text { de ítems }\end{array}$ \\
\hline $\begin{array}{l}\text { Cuestionario } \\
\text { de Convivencia } \\
\text { Escolar }\end{array}$ & $\begin{array}{l}\text { Nicolás, } \\
2015\end{array}$ & $\begin{array}{l}\text { "Convivencia y } \\
\text { Satisfacción en } \\
\text { la institución } \\
\text { educativa" } \\
\text { "Comportamientos } \\
\text { que pueden } \\
\text { generar conflictos } \\
\text { en la convivencia } \\
\text { escolar" } \\
\text { "Resolución de } \\
\text { conflictos en } \\
\text { la convivencia } \\
\text { escolar" }\end{array}$ & $\begin{array}{l}13 \\
10 \\
7\end{array}$ \\
\hline $\begin{array}{l}\text { Modos de } \\
\text { Manejo de } \\
\text { Conflicto }\end{array}$ & $\begin{array}{l}\text { Thomas y } \\
\text { Kilmann, } \\
2014\end{array}$ & $\begin{array}{l}\text { Asertividad } \\
\text { Cooperación }\end{array}$ & $\begin{array}{l}15 \\
15\end{array}$ \\
\hline $\begin{array}{c}\text { Escala de } \\
\text { dificultades en } \\
\text { la regulación } \\
\text { emocional } \\
\text { (DERS-español) }\end{array}$ & $\begin{array}{l}\text { Marín, } \\
\text { Robles, } \\
\text { González } \\
\text { y Andrade, } \\
\mathbf{2 0 1 2 .}\end{array}$ & $\begin{array}{l}\text { Capacidad de } \\
\text { regulación de } \\
\text { estados afectivos }\end{array}$ & 8 \\
\hline $\begin{array}{c}\text { Encuesta sobre } \\
\text { estilos de } \\
\text { liderazgo }\end{array}$ & $\begin{array}{l}\text { Castillo, } \\
\text { 2010, en } \\
\text { Reyes, } \\
2012 .\end{array}$ & $\begin{array}{c}\text { Procesos } \\
\text { decisorios. } \\
\text { Sistema de } \\
\text { comunicación. } \\
\text { Relaciones } \\
\text { interpersonales. } \\
\text { Sistemas de } \\
\text { recompensas y } \\
\text { castigos. }\end{array}$ & $\begin{array}{l}5 \\
5 \\
5 \\
5\end{array}$ \\
\hline
\end{tabular}

Además, se ofrecen dos manuales de trabajo, uno para el estudiante, donde se explica, de manera detallada, el contenido informativo relacionado a las habilidades que se pretende desarrolla a lo largo de la implementación del programa, reforzando el aprendizaje. El otro se diseñó para los instructores, explicando la lógica de cada secuencia didáctica, ofreciendo una guía para ubicar la información en cada presentación que se utiliza, así como la forma de implementación de cada actividad diseñada.

\section{Conclusiones}

Construir ciudadanía, desde una propuesta de un programa educativo innovador, que sume a la formación de los estudiantes universitarios hacía horizontes distintos a los especificados en los planes de estudio, abona a la idea de generar una sociedad más crítica, comprensiva, empática, solidaría y colaborativa, en donde los profesionales mantengan una mejor conciencia social sobre su impacto en la comunidad donde ellos se desarrollaron.

Tomar en consideración la convivencia, dentro de la lógica de la Formación Ciudadana, permite entender la manera en que los ciudadanos pueden sentirse identificados con su comunidad, y así promover la unión para lograr objetivos en común que beneficien a cada uno de ellos. Además, de enfatizar que las situaciones conflictivas siempre estarán presentes en su interacción cotidiana, volviendo relevante el aprendizaje de alternativas que se enfoquen en la colaboración, más que en la competitividad.

Los resultados de la fundamentación del programa muestran la relevancia de reforzar en los estudiantes universitarios, el conjunto de habilidades emocionales para resolver conflictos los cuales como establece Chaux (2012) son pilares para la construcción de la formación en ciudadanía.

Aun cuando son básicos, no son los únicos para la construcción de la ciudadanía, dado que los estudiantes reportaron dificultades para que en su institución hubiera procedimientos claros para resolver conflictos en otras instancias, cuando no es posible llegar a acuerdos, por lo que no solo es trabajar en el individuo, sino en las instituciones para crear espacios de convivencia y solución de conflictos a través de normas y protocolos claros de atención, como lo mencionan Mejía y Perafán (2006). Por tanto, las instituciones deben tener espacios mediadores para que los actores de las instituciones acudan a ellas en el caso de requerirlos.

Por ello, la inclusión de programas educativos innovadores que apoyen a concretar esta idea en los estudiantes universitarios corresponde a un avance significativo a la manera en que se puede construir diversos espacios en las Instituciones de Educación Superior que ofrezcan las suficientes condiciones para el diálogo, la reflexión, la participación, y el involucramiento de los individuos en su comunidad universitaria.

La propuesta presentada en este trabajo fue diseñada para cumplir ese propósito, pues cada sesión de trabajo se estructuró para que el estudiante fuera un agente activo al construir su propio aprendizaje, tomando en consideración su participación constante, de manera individual y en colaboración con sus compañeros de aula, a través de juegos, 
estudios de caso, creación de foros o talleres, entre otros aspectos, considerándose como un colega. Con ello, se aporta una propuesta fundamentada en principios, competencias y métodos congruentes para la promoción de la ciudadanía en el ámbito de cultura de paz.

Por lo tanto, ofrecer estos espacios en forma de talleres extracurriculares, o como parte oficial de los planes de estudio de cada licenciatura, coadyuvará a la consecución de los objetivos institucionales de cada IES, ajustándose también a las recomendaciones internacionales y nacionales, sobre las transformaciones que habría que tomarse en cuenta para la educación, yendo más allá del umbral tradicional que ha permeado durante mucho tiempo en el sistema educativo.

\section{Referencias bibliográficas}

Andrés, M., Labrador, M. (2014). Aprender con los demás y mejorar las competencias comunicativas a través del foro. Lenguaje y textos, 40, 87-96.

Aparicio, O., Ostos, O. (2018). El constructivismo y el construccionismo. Revista Interamericana de Investigación, Educación y Pedagogía, 11(2), 115-120.

Barrios, A. (2020). El baúl de la paz sistematización de una práctica pedagógica de la cátedra de la paz. Universidad de Antioquia. Medellín, Colombia. (Tesis de Maestría). http://bibliotecadigital.udea.edu. co/bitstream/10495/16404/2/BarriosArelis_2020_ BaulPazSistematizacion.pdf

Betancourt, R., Guevara, L., Fuentes, E. (2011). El taller como estrategia didáctica, sus fases y componentes para el desarrollo de un proceso de cualificación en el uso de tecnologías de la información y la comunicación (TIC) con docentes de lenguas extranjeras. Caracterización y Retos.

Carreño, M. Rozo, H. (2020). Estrategias para desarrollar la convivencia y la paz desde la educación. Academia y virtualidad,13(2), 35-56. DOI: 10.18359/ravi.4501

Chaux, E. (2012). Educación, convivencia y agresión escolar. Bogotá: Distribuidora y Editora Aguilar

Chávez, D. (2021). ConVive: Una propuesta de programa para la formación de habilidades de resolución de conflictos en estudiantes universitarios. Tesis de maestría, Universidad de Sonora.

Cresswell, J. (2009). Research design. Qualitative, quantitive and mixed methods approaches. Editorial: Sage.

Hamra, S. (2020). Estudios generales y las tecnologías de la información y la comunicación en la formación ciudadana de los estudiantes universitarios. Revista Ciencias de la Educación, 30(46), 630-662.

Holguín, E. (2018). Técnica del sociodrama en la comprensión lectora en el subnivel elemental. Tesis. Facultad de Filosofía, Letras y Ciencias de la Educación.

Iborra, A., e Izquierdo, M. (2010). ¿Cómo afrontar la evaluación del aprendizaje colaborativo? Una propuesta valorando el proceso, contenido y el producto de la actividad grupal. Revista General de Información y Documentación, 20, 221-241.

Mejía, A., y Perafán, B. (2006). Para acercarse al dragón, para amansarlo, es necesario haberlo amansado primero: Una mirada crítica a las competencias ciudadanas. Revista de Estudios Sociales, (23), 23-35.

León, F. (2020). Competencias ciudadanas: análisis crítico y pautas para la formación profesional. Controversias y concurrencias Latinoamericanas, 12(21), 369-388.

Marín, M., Robles, R., González-Forteza, C., Andrade, P. (2012). Propiedades psicométricas de la escala "Dificultades en la Regulación Emocional" en español (DERS-E) para adolescentes mexicanos. Salud mental, 35(6), 521- 526.

Martínez, M. (20026). Formación para la ciudadanía y educación superior. Revista Iberoamericana de la Educación, 42, 85-102. https://rieoei.org/historico/ documentos/rie42a05.pdf

Mescua, Ramos, Ramírez, Cruz, Caycho (2020). Niveles de competencias ciudadanas en estudiantes universitarios de Ciencias de la Salud. Revista EDUSER, 7(1) 69-81. http://181.224.246.204/index. php/EDUSER/article/view/2516/2069

Ministerio de Educación Nacional. (2011). Orientaciones para la Institucionalización de las competencias ciudadanas. Cartilla Brújula 1: Programa de competencias ciudadanas. Bogotá. D.C., Colombia: Amado Impresiones S.A.S.

Montoya, J. (2008). Segundo avance de investigación - El desarrollo de competencias ciudadanas en el ámbito escolar. Revista Virtual Universidad Católica del Norte, (24). https://www.redalyc.org/ pdf/1942/194220359004.pdf

Murillo Castaño, Gabriel, \& Castañeda Aponte, Nathalia (2007). Competencias ciudadanas y construcción de ciudadanía juvenil. Revista del CLAD Reforma y Democracia, (37),1-17. https://www.redalyc.org/ articulo.oa?id=35753366900 5

Nicolás, A. (2015). La convivencia escolar en los centros de Educación Secundaria de la Región de Murcia: La voz del alumnado. Tesis doctoral. Departamento de didáctica y organización escolar. Universidad de Murcia. 
Ortiz, E., Hincapié, D. (2019). El desarrollo de las habilidades socioemocionales en los sistemas educativos de América Latina. En Mateo, M., y Rucci, G. (2019) El futuro ya está aquí. Habilidades transversales en América Latina y el Caribe en el siglo XXI. Editorial: Banco Interamericano de Desarrollo.

Ortiz, G. y Lemus, M. (2020). Ciudadanía y participación juvenil en la Universidad Autónoma Metropolitana. En: Formación Ciudadana en Estudiantes Universitarios, Editorial: Terracota, 53-79.

Padilla, N. (2011). Metodología para el diseño de videojuegos educativos sobre una arquitectura para el análisis del aprendizaje colaborativo. Granada: Universidad de Granada.

Pinilla, N.y Mendieta, M. (2017). Pedagogía dela confianza: una estrategia para generar ambientes escolares de paz. Revista Análisis, 49(91), 315-336. https://www.redalyc. org/journal/5155/515558054004/515558054004.pdf

Reyes, N. (2012). Liderazgo directivoy desempeño docente en el nivel secundario de una institución educativa de Ventanilla-Callao. Tesis de maestría. Universidad San Ignacio de Loyola.

Ruiz, A. y Chaux, E. (2005). La formación de competencias ciudadanas. https://appsciso.uniandes.edu.co/sip/ data/pdf/formacion_de_competencias_ciudadanas. pdf

Schunk, D. (2009). Teorías del aprendizaje. Una perspectiva educativa. Editorial: Pearson, pp. 228-278.
Scagnoli, N. (2005). El aprendizaje colaborativo en cursos a distancia. Investigación y Ciencia, 14(36), 39-47.

Tamayo, M. (2011). El proceso de la investigación científica. Editorial: Limusa. Pp. 44-50.

Thomas, K., Kilmann, R. (2014). Instrumento ThomasKilmann de modos de conflicto. Editorial: CPP.

Torrecillas, E., Olmos, S., Rodríguez, M., Martínez, F. (2016). Eficacia de un programa de formación de profesorado de Educación Secundaria sobre resolución de conflictos, con apoyo tecnológico. Digital Education.

Urbina, M., Medina, S., Calle, C. (2010). Herramientas para el aprendizaje colaborativo: una aplicación del juego de rol. Teoría de la educación: educación y cultura en la sociedad de la información, 11(3), 277300.

Verdeja, M. (2013). Aprendizaje de la convivencia en contextos educativos: identificando problemas y formulando propuestas de mejora desde la acción tutorial. Revista latinoamericana de educación inclusiva, 6(2), 167-190.

Venet Muñoz, R. (2019). La formación ciudadana en el contexto universitario. Una mirada axiológicocultural desde la educación para la pazy la noviolencia. Revista Conrado, 15(70), 435-443. Recuperado de http://conrado.ucf. edu.cu/index.php/Conrad

Zuta, E.; Velasco, A. y Rodríguez, J. (2014). Desarrollo de competencias ciudadanas mediante un curso socialmente responsable. Educación, 6, 51-66. 